\title{
Nitric oxide, afferent sensory nerves, and antioxidative enzymes in the mechanism of protection mediated by tricarbonyldichlororuthenium(II) dimer and sodium hydrosulfide against aspirin-induced gastric damage
}

\author{
Marcin Magierowski $^{1} \cdot$ Magdalena Hubalewska-Mazgaj $^{2} \cdot$ Katarzyna Magierowska $^{1} \cdot$ \\ Dagmara Wojcik $^{1} \cdot$ Zbigniew Sliwowski $^{1} \cdot$ Slawomir Kwiecien $^{1} \cdot$ Tomasz Brzozowski $^{1}$
}

Received: 1 December 2016/Accepted: 10 February 2017/Published online: 25 February 2017

(c) The Author(s) 2017. This article is published with open access at Springerlink.com

\begin{abstract}
Background Aspirin exerts side effects within the gastrointestinal tract. Hydrogen sulfide $\left(\mathrm{H}_{2} \mathrm{~S}\right)$ and carbon monoxide $(\mathrm{CO})$ have been implicated in gastroprotection but the mechanism of beneficial action of these gaseous mediators against aspirin-induced damage has not been fully studied. We determined the involvement of afferent sensory neurons, calcitonin-gene-related peptide (CGRP), lipid peroxidation, and nitric oxide (NO) biosynthesis in gastroprotection of $\mathrm{H}_{2} \mathrm{~S}$-releasing $\mathrm{NaHS}$ and CO-releasing tricarbonyldichlororuthenium(II) dimer (CORM-2) against aspirin-induced injury.

Methods Wistar rats with or without capsaicin-induced denervation of sensory neurons were pretreated with vehicle, CORM-2 (5 mg/kg intragastrically), or NaHS (5 mg/kg intragastrically) with or without capsazepine $\left(5 \mathrm{mg} / \mathrm{kg}\right.$ intragastrically) or $N^{\mathrm{G}}$-nitro-L-arginine (L-NNA, $20 \mathrm{mg} / \mathrm{kg}$ intraperitoneally). The areas of aspirin-induced lesions and gastric blood flow (GBF) were assessed by planimetry and laser flowmetry respectively. Gastric mucosal messenger RNA and/or protein expression of CGRP, heme oxygenase 1, inducible nitric oxide synthase, cyclooxygenase 2 , interleukin- $1 \beta$, glutathione peroxidase 1 (GPx-1), and superoxide dismutase was determined by real-time PCR or Western blot. Malondialdehyde
\end{abstract}

Marcin Magierowski

m.magierowski@uj.edu.pl

1 Department of Physiology, Jagiellonian University Medical College, 16 Grzegorzecka Street, 31-531 Cracow, Poland

2 Department of Genetic Research and Nutrigenomics, Malopolska Centre of Biotechnology, Jagiellonian University, 7A Gronostajowa Street, 30-387 Cracow, Poland
(MDA) and 4-hydroxynonenal (4-HNE) content was determined by colorimetric assay.

Results Aspirin caused gastric lesions, decreased GBF, and raised MDA content, but pretreatment with NaHS and CORM-2 reduced these effects. Capsaicin-induced denervation or co-treatment with capsazepine reversed the gastroprotective and vasodilatory effects of NaHS but not those of CORM-2. L-NNA reversed NaHS-induced gastroprotection and partly reduced CORM-2-induced gastroprotection. NaHS and CORM-2 decreased MDA and 4-HNE content, restoring GPx-1 protein expression.

Conclusions We conclude that $\mathrm{H}_{2} \mathrm{~S}$ - but not CO-mediated gastroprotection against aspirin-induced injury involves afferent sensory nerves and partly NO activity. NaHS and CORM-2 prevented aspirin-induced gastric mucosal lipid peroxidation via restoration of microcirculation and antioxidative GPx-1 protein expression.

Keywords Hydrogen sulfide - Carbon monoxide - Nitric oxide $\cdot$ Afferent sensory nerves $\cdot$ Aspirin

\section{Introduction}

Aspirin is the most popular representative among the nonsteroidal anti-inflammatory drugs (NSAIDs), which, however, exerts side effects within the gastrointestinal tract, including hemorrhagic microbleedings and gastric erosions [1-4]. The gaseous mediators hydrogen sulfide $\left(\mathrm{H}_{2} \mathrm{~S}\right)$, carbon monoxide $(\mathrm{CO})$, and nitric oxide $(\mathrm{NO})$ were reported to afford gastroprotection against injury induced by noxious agents but the mechanism of this beneficial action of these molecules has not been fully elucidated [5-10]. All three mediators have been shown to take part in 
regulation of physiological processes in the gastrointestinal tract and other systems [11-13].

The $\mathrm{H}_{2} \mathrm{~S}$-releasing derivatives of NSAIDs, such as ATB-346, exert an anti-inflammatory effect similar to that of the parent drugs (naproxen in the case of ATB346) but cause pronouncedly fewer side effects as compared with the native form of the drug. This indicates the usefulness of this new class of $\mathrm{H}_{2} \mathrm{~S}$ derivatives of NSAIDs and the potential safety and possible translation to clinical settings [14]. Moreover, these recently introduced NSAID prodrugs-namely, $\mathrm{H}_{2} \mathrm{~S}$-releasing anti-inflammatory drugs-were suggested as promising agents in chemoprevention of cancers [15, 16]. Recently, it has been reported that inhibition of $\mathrm{H}_{2} \mathrm{~S}$ generation contributes to the formation of aspirin-induced gastric injury [5]. The $\mathrm{CO}$ and $\mathrm{H}_{2} \mathrm{~S}$ biosynthesis pathways were both shown to synergistically interact in the mechanism of gastroprotection against NSAID-induced gastric damage via restoration of gastric microcirculation impaired by these drugs [17].

We aimed to investigate the involvement of afferent sensory neurons, calcitonin-gene-related peptide (CGRP), and vanilloid receptor 1 (also known as transient receptor potential cation channel V1; TRPV1) in gastroprotection of $\mathrm{H}_{2} \mathrm{~S}$ released from NaHS and $\mathrm{CO}$ released from tricarbonyldichlororuthenium(II) dimer (CORM-2) and the accompanying changes in gastric blood flow (GBF) with regard to experimental gastric lesions induced by aspirin. Moreover, we studied the potential role of $\mathrm{H}_{2} \mathrm{~S}$ and $\mathrm{CO}$ in regulation of aspirin-induced lipid peroxidation. We also investigated the involvement of endogenous $\mathrm{NO}$ and messenger RNA (mRNA) or protein expression of the antioxidizing enzymes glutathione peroxidase 1 (GPx-1) and superoxide dismutase (SOD) in the protective activity of $\mathrm{H}_{2} \mathrm{~S}$ and $\mathrm{CO}$ against aspirin-induced ulcerogenesis.

\section{Materials and methods}

\section{Animals, chemicals and drugs, and determination of the gastric damage and GBF}

Seventy male Wistar rats weighting 220-300 g were fasted for $24 \mathrm{~h}$ with free access to drinking water before the experiments. The study was approved by the Institutional Animal Care and Use Committee of Jagiellonian University Medical College in Cracow and was conducted in accordance with the Helsinki Declaration regarding handling of experimental animals.

Two weeks before the experiment, capsaicin was administered subcutaneously to some of the rats (series A) in three doses of 25,50 , and $50 \mathrm{mg} / \mathrm{kg}$ (total dose $125 \mathrm{mg} /$ $\mathrm{kg}$ ) to induce the functional ablation of sensory nerves as reported in our previous studies [18, 19].

On the day of experiment, aspirin dissolved in $0.2 \mathrm{M}$ $\mathrm{HCl}$ solution was administered intragastrically in a dose of $125 \mathrm{mg} / \mathrm{kg}$ ( $1.5 \mathrm{ml}$ per rat) to induce gastric lesions, as described previously [17]. Thirty minutes before aspirin application, rats with capsaicin-induced denervation (series A) and rats without capsaicin-induced denervation (series B) were pretreated intragastrically with (1) $0.9 \%$ saline (vehicle control) or dimethyl sulfoxide and saline (1:10), (2) CO-releasing CORM-2 [20], applied in a dose of $5 \mathrm{mg} /$ $\mathrm{kg}$, which has previously been reported by our group to increase $\mathrm{CO}$ content in gastric mucosa and to exert gastroprotection against aspirin- and ethanol-induced gastric damage $[17,21]$, or (3) NaHS, the $\mathrm{H}_{2} \mathrm{~S}$-releasing salt applied intragastrically in a dose of $5 \mathrm{mg} / \mathrm{kg}$, which has been demonstrated to protect gastric mucosa against aspirin-, stress-, or alendronate-induced lesions $[17,19,22]$. In a separate series (C), rats with intact sensory nerves were pretreated with NaHS or CORM-2 in combination with capsazepine ( $5 \mathrm{mg} / \mathrm{kg}$ intragastrically), a TRPV1 antagonist [23], or $N^{\mathrm{G}}$-nitro-L-arginine (L-NNA, $20 \mathrm{mg} / \mathrm{kg}$ intraperitoneally), a nonselective nitric oxide synthase (NOS) inhibitor [24]. All chemicals were purchased from Sigma-Aldrich (Schnelldorf, Germany) and were injected intraperitoneally or administered intragastrically by means of an orogastric tube as reported previously $[18,25]$.

One hour after administration of aspirin, rats were anesthetized with pentobarbital $(60 \mathrm{mg} / \mathrm{kg}$ intraperitoneally). Their abdomens were opened for the GBF measurement by a laser Doppler flowmeter (Laserflo blood perfusion monitor, model BPM 403A, Vasamedics, St Paul, MN, USA), as described elsewhere [25]. The stomach was excised and opened along the greater curvature to determine the area of aspirin-induced gastric lesions by means of computerized planimetry (Morphomat, Carl Zeiss, Berlin, Germany) [26, 27]. Next, the gastric mucosal samples from oxyntic mucosa were scraped off on ice, snap-frozen in liquid nitrogen, and stored at $-80{ }^{\circ} \mathrm{C}$ until further analysis [21].

\section{Determination of mRNA expression of $\beta$-actin, $\alpha$ - CGRP, heme oxygenase 1 , cyclooxygenase 2 , inducible NOS, interleukin-1ß, GPx-1, and SOD-2 in rat gastric mucosa by real-time polymerase chain reaction}

Expression of mRNA for particular genes of interest in gastric mucosa was determined by real-time polymerase chain reaction (PCR) as described previously [21, 22]. Briefly, RNA was isolated from gastric mucosal biopsy samples with use of a GeneMATRIX universal RNA 
purification kit (EURx, Gdansk, Poland). Reverse transcription to complementary DNA was performed with a high-capacity complementary DNA reverse transcription kit (Thermo Fisher Scientific, Life Technologies, MA, USA). Expression of $\beta$-actin, heme oxygenase 1 (HO-1), cyclooxygenase (COX)-2, inducible NOS (iNOS), interleukin-1 $\beta$ (IL-1 $\beta$ ), GPx-1, and SOD-2 was determined by real-time PCR using specific primers [17, 22], SG qPCR master mix $(2 \times)$ including SYBR Green (EURx, Gdansk, Poland), and Quant Studio 12K Flex thermal cycler (Thermo Fisher Scientific, MA, USA). To determine $\alpha$ CGRP mRNA expression, 5'-GCTCACCAGGGAGGCATCAT- $3^{\prime}$ forward primer and $5^{\prime}$-ATGCCTGGTACAGGAGCAAGA- $3^{\prime}$ reverse primer were used. $\beta$-Actin was used as an internal control. Data were analyzed by the $2^{-\Delta C_{\mathrm{t}}}$ method [28].

\section{Determination of protein expression in gastric mucosa by Western blot}

Protein expression of $\beta$-actin, GPx-1, SOD- 1 , and iNOS in gastric mucosa was determined by Western blot, as described previously [17, 21, 22]. Rabbit polyclonal antiiNOS antibody (1:500, ab3523, Abcam, Cambridge, UK), rabbit polyclonal anti-GPx-1 antibody (1:1000, ab22604, Abcam), rabbit polyclonal anti-SOD-1 antibody (1:1000, ab13498, Abcam), and rabbit polyclonal anti- $\beta$-actin anitbody (1:1000, 20536-1-AP, Proteintech, Manchester, UK) were used as primary antibodies. Protein expression was visualized by means of peroxidase-conjugated secondary goat anti-rabbit IgG antibody (sc-2004, Santa Cruz Biotechnology) at a dilution of 1:2000. All primary and secondary antibodies were diluted in 5\% nonfat milk.

Chemiluminescence was developed with use of WesternSure ${ }^{\circledR}$ enhanced chemiluminescence substrate (LICOR, Lincoln, NE, USA) and was measured with a C-DiGit ${ }^{\circledR}$ blot scanner (LI-COR). The intensity of bands was determined and analyzed by Image Studio 4.0 (LICOR). The expression of the proteins of interest was normalized to the expression of $\beta$-actin as a loading control.

\section{Determination of the gastric mucosal lipid peroxidation as malondialdehyde and 4- hydroxynonenal content}

To determine malondialdehyde (MDA) and 4-hydroxynonenal (4-HNE) tissue concentration, the colorimetric assay for lipid peroxidation (Bioxytech LPO-586, Oxis, Portland, OR, USA) was used as described previously [27]. In brief, about $200 \mathrm{mg}$ of gastric mucosa was excised and then quickly washed in a test tube, and $20 \mu \mathrm{L} 0.5 \mathrm{M}$ butylated hydroxytoluene was added to prevent sample oxidation. Next, samples were homogenized in $20 \mathrm{mM}$ tris(hydroxymethyl)aminomethane for $15 \mathrm{~s}$ in $\mathrm{pH} 7.4$ and centrifuged (3000 $\mathrm{g}$ at $4{ }^{\circ} \mathrm{C}$ for $10 \mathrm{~min}$ ). The supernatant was collected and stored at $-80{ }^{\circ} \mathrm{C}$ until further analysis. The colorimetric assay used to determine MDA concentration in gastric mucosa is based on the reaction of a chromogenic reagent ( $N$-methyl-2-phenylindole) with MDA and 4-HNE at $45^{\circ} \mathrm{C}$, which yields a stable chromophore with maximal absorbance at $586 \mathrm{~nm}$, analyzed with a microplate reader (Tecan Sunrise, Männedorf, Switzerland).

\section{Statistical analysis}

The results are presented as the mean \pm standard error of the mean. Statistical analysis was performed with Student's $t$ test or the Mann-Whitney $U$ test if appropriate assumptions did not hold. ANOVA with the Tukey post hoc test was used if more than two experimental groups were compared. We considered $p<0.05$ to be statistically significant. Data were analyzed with GraphPad Prism 5.0.

\section{Results}

\section{Effect of afferent sensory nerve ablation and inhibition of TRPV1 receptors on CORM-2- and NaHS-mediated gastroprotection}

Figure 1 shows that pretreatment with CORM-2 $(5 \mathrm{mg} / \mathrm{kg}$ intragastrically) and NaHS (5 mg/kg intragastrically) significantly decreased the mean area of gastric lesions and significantly increased GBF as compared with vehicle pretreatment $(p<0.05)$ (Fig. 1). These data confirm our previously published results that $\mathrm{CO}$ and $\mathrm{H}_{2} \mathrm{~S}$ donors protected gastric mucosa against aspirin-induced gastric damage [17]. Capsaicin-induced denervation significantly increased the area of aspirin-induced gastric lesions and decreased GBF as compared with vehicle treatment in rats with intact sensory nerves $(p<0.05)$ (Fig. 1). The reduction of the area of gastric lesions and the accompanying increase in GBF caused by NaHS were reversed in rats with capsaicin-induced denervation $(p<0.05)$ (Fig. 1). Capsazepine $(5 \mathrm{mg} / \mathrm{kg}$ intragastrically) by itself did not affect aspirin-induced gastric damage and GBF as compared with vehicle treatment (Fig. 1). When NaHS was combined with capsazepine, a significant increase in the mean area of gastric lesions as compared with that for rats pretreated with this $\mathrm{H}_{2} \mathrm{~S}$ donor alone was observed $(p<0.05)$, whereas GBF was significantly increased as compared with that in the vehicle-control group $(p<0.05)$. The capsazepine and CORM- 2 co-treatment failed to affect the decrease of gastric damage area and the increase in GBF as compared with the respective values obtained in the group of rats pretreated with CORM-2 alone (Fig. 1). 

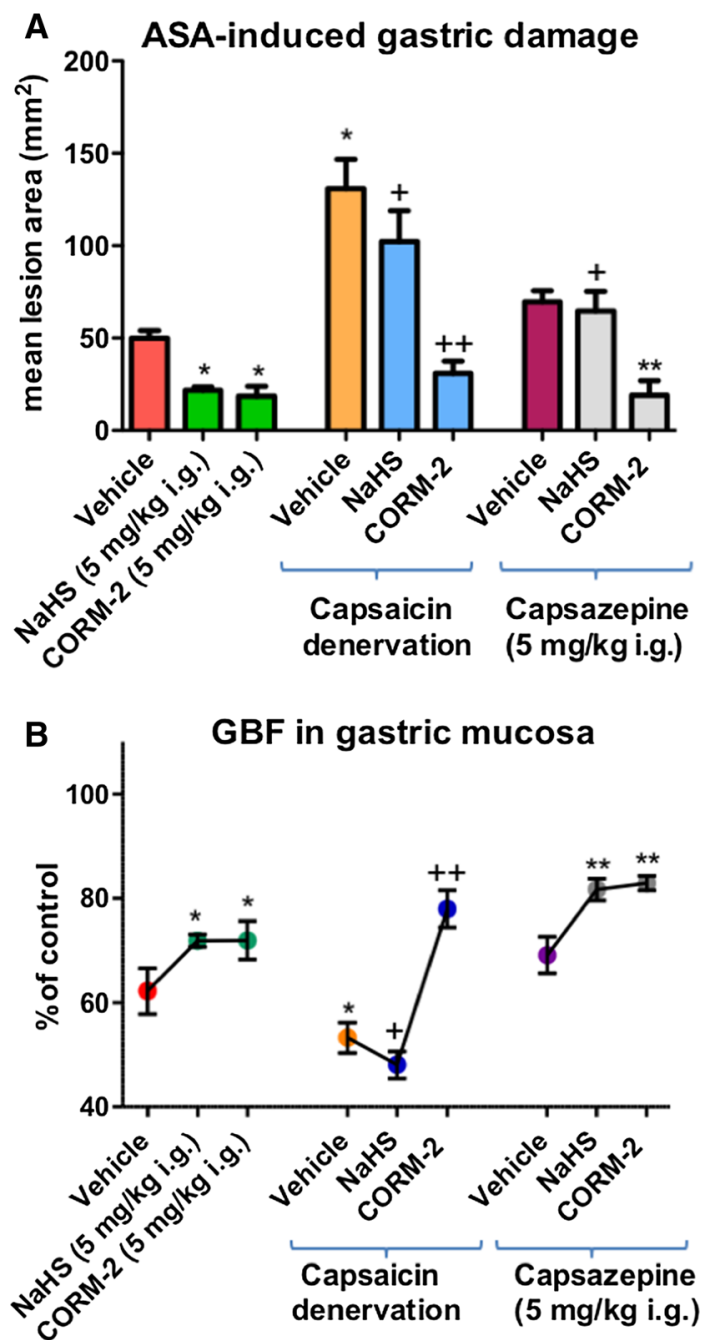

Fig. 1 a Mean lesion area and $\mathbf{b}$ gastric blood flow $(G B F)$ in gastric mucosa of rats with or without afferent sensory nerves denervation and pretreated with vehicle, NaHS $(5 \mathrm{mg} / \mathrm{kg}$ intragastrically), or tricarbonyldichlororuthenium(II) dimer (CORM-2;5 mg/kg intragastrically) alone or in combination with capsazepine $(5 \mathrm{mg} / \mathrm{kg}$ intragastrically) and administered with aspirin (ASA; $125 \mathrm{mg} / \mathrm{kg}$ intragastrically). The results are mean \pm standard error of the mean for four or five rats per experimental group. A significant change as compared with the respective values in the vehicle-control group with intact sensory nerves is indicated by one asterisk $(p<0.05)$. One cross indicates a significant change as compared with the respective values in rats with intact sensory neurons and pretreated with NaHS alone $(p<0.05)$. A significant change as compared with the respective values in the capsaicin-denervated vehicle-control group is indicated by two crosses $(p<0.05)$. A significant change as compared with the respective values in the vehicle-control group treated with capsazepine alone is indicated by two asterisks $(p<0.05)$. i.g. intragastrically

\section{Expression of CGRP mRNA in gastric mucosa}

Figure 2 shows that the CGRP mRNA expression in gastric mucosa with aspirin-induced gastric damage was significantly increased in rats with capsaicin-induced denervation pretreated with vehicle as compared with the respective

\section{CGRP mRNA expression in gastric mucosa}

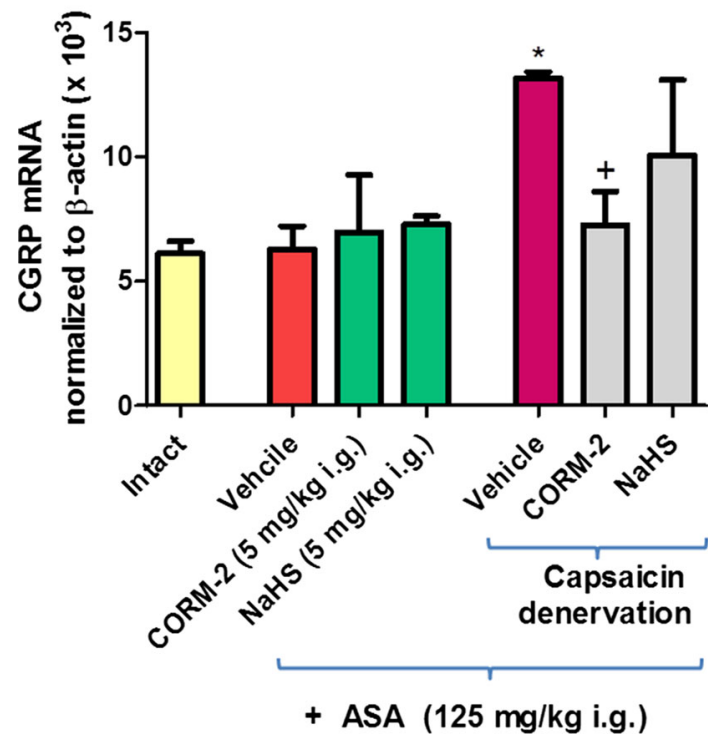

Fig. 2 Calcitonin-gene-related peptide (CGRP) messenger RNA ( $m R N A)$ expression in gastric mucosa of rats with or without capsaicin-induced denervation and pretreated with vehicle, NaHS ( $5 \mathrm{mg} / \mathrm{kg}$ intragastrically), or tricarbonyldichlororuthenium(II) dimer (CORM-2; $5 \mathrm{mg} / \mathrm{kg}$ intragastrically) and administered with aspirin ( $A S A ; 125 \mathrm{mg} / \mathrm{kg}$ intragastrically). Intact refers to healthy rats without any treatment. The results are expressed as CGRP mRNA expression normalized to $\beta$-actin expression and are the mean \pm standard error of the mean for four or five rats per group. An asterisk indicates a significant change as compared with the values in the vehicle-control group without capsaicin-induced denervation $(p<0.05)$. A cross indicates a significant change as compared with the values obtained in the vehicle-pretreated group with capsaicin-induced denervation $(p<0.05)$. i.g. intragastrically

values in intact gastric mucosa and the vehicle-pretreated group without sensory denervation $(p<0.05)$ (Fig. 2). Pretreatment with CORM-2 but not with NaHS significantly decreased mRNA expression of CGRP in gastric mucosa of rats with capsaicin-induced functional ablation of afferent sensory neurons in which aspirin was adminsitered as compared with the vehicle-control group $(p<0.05)$ (Fig. 2).

\section{Expression of HO-1, iNOS, COX-2, and IL-1及 mRNA in gastric mucosa}

Figure 3 shows that the mRNA expression for HO-1, iNOS, COX-2, or IL-1 $\beta$ was significantly increased in vehicle-pretreated gastric mucosa in rats administered with aspirin as compared with that observed in intact rats $(p<0.05)$. Pretreatment with CORM-2 significantly increased mRNA expression of HO-1 and significantly decreased mRNA expression of iNOS, COX-2, and IL- $1 \beta$ in rats with aspirin-induced gastric damage as compared with the vehicle-control group without capsaicin-induced 

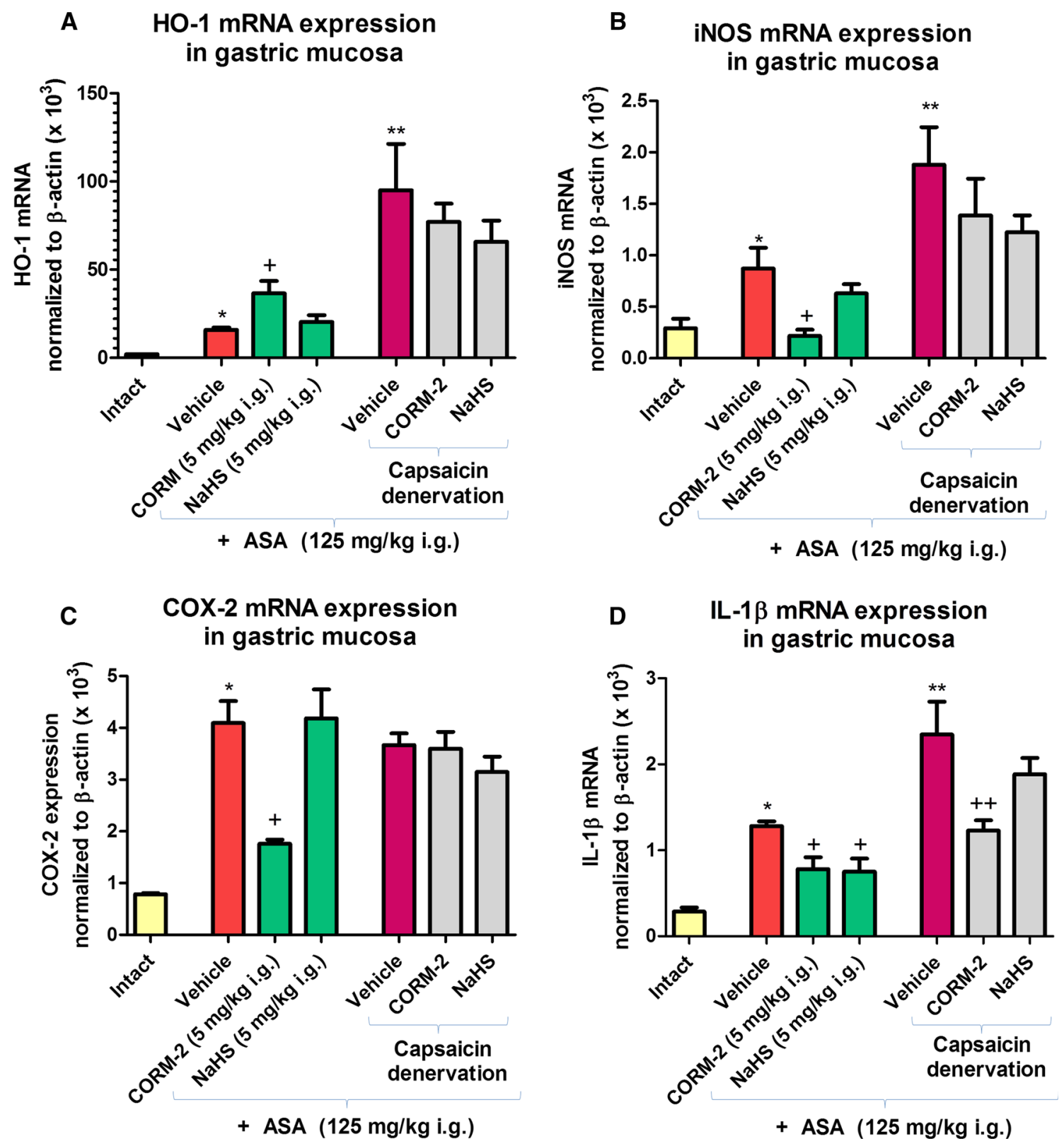

Fig. 3 Messenger RNA ( $m R N A$ ) expression of $a$ heme oxygenase 1 $(H O-1), \mathbf{b}$ inducible nitric oxide synthase (iNOS), c cyclooxygenase 2 $(C O X-2)$, and $\mathbf{d}$ interleukin-1 $\beta(I L-1 \beta)$ in gastric mucosa of rats with or without capsaicin-induced denervation and pretreated with vehicle, $\mathrm{NaHS}$ ( $5 \mathrm{mg} / \mathrm{kg}$ intragastrically), or tricarbonyldichlororuthenium(II) dimer (CORM-2; $5 \mathrm{mg} / \mathrm{kg}$ intragastrically) and $30 \mathrm{~min}$ later given aspirin $(A S A ; 125 \mathrm{mg} / \mathrm{kg}$ intragastrically). Intact refers to healthy rats without any treatment. The results are expressed as mRNA expression of HO-1 (a), iNOS (b), COX-2 (c), and IL-1 $\beta$ (d) normalized to $\beta$ -

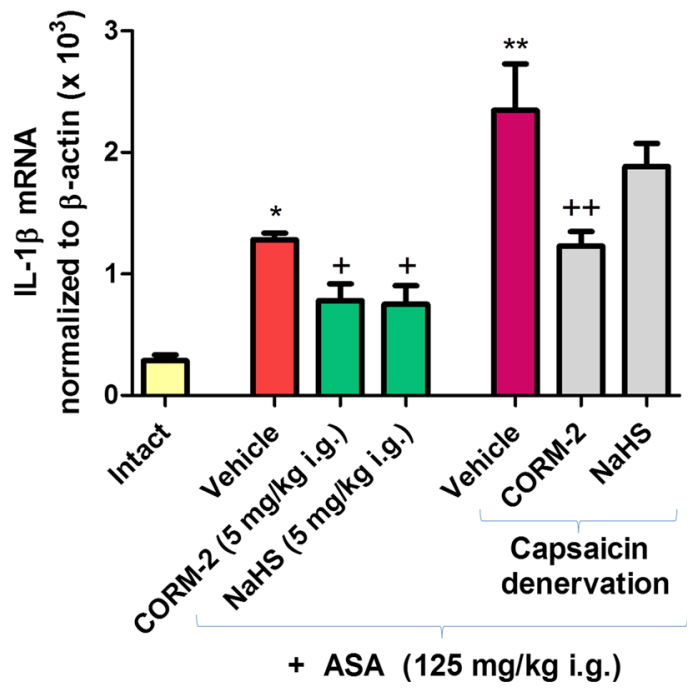

actin expression and are the mean \pm standard error of the mean of four or five determinations per group. One asterisk indicates a significant change as compared with the values in intact gastric mucosa $(p<0.05)$. A significant change as compared with the values obtained in the vehicle-pretreated group with intact sensory nerves is indicated by a cross or two asterisks $(p<0.05)$. A significant change as compared with the vehicle-control group with capsaicin-induced denervation is indicated by two crosses $(p<0.05)$. i.g. intragastrically

denervation $(p<0.05)$ (Fig. 3). The pretreatment with NaHS significantly decreased mRNA expression of IL-1 $\beta$ but not that of HO-1, iNOS, or COX-2 as compared with vehicle treatment without capsaicin-induced denervation $(p<0.05)$ (Fig. 3). These results confirmed our data published previously [17]. In vehicle-pretreated rats with capsaicin-induced denervation, mRNA expression of HO-

1 , iNOS, or IL-1 $\beta$ was significantly increased as compared with that in respective group without capsaicin denervation $(p<0.05)$ (Fig. 3a, b, d). In rats with afferent sensory nerves ablation, pretreatment with CORM-2 but not NaHS significantly decreased IL- $1 \beta$ mRNA expression as compared with that in the vehicle-treated control group $(p<0.05)$ (Fig. 3d). Pretreatment with CORM-2 or NaHS 
did not affect mRNA expression of HO-1, iNOS, or COX-2 in gastric mucosa of rats with sensory nerve ablation and given aspirin.

\section{Expression of GPx-1 and SOD-2 mRNA in gastric mucosa}

The results for gastric mucosal mRNA expression of GPx-1 and SOD-2 in intact rats and those with or without capsaicin-induced denervation pretreated with vehicle, CORM-2, or NaHS and exposed to aspirin are presented in Fig. 4. The expression of GPX-1 and SOD-2 mRNA was significantly decreased in vehicle-pretreated rats given aspirin as compared with intact rats $(p<0.05)$. Pretreatment with NaHS significantly increased GPx-1 and SOD-2 mRNA expression in gastric mucosa of rats with intact sensory nerves $(p<0.05)$ (Fig. 4). In rats with intact sensory nerves, the pretreatment with NaHS failed to affect GPx-1 or SOD-2 mRNA expression as compared with vehicle pretreatment combined with exposure to aspirin (Fig. 4). Likewise, in rats with capsaicin-induced denervation, the pretreatment with NaHS did not affect GPx-1 or SOD-2 mRNA expression as compared with vehicle pretreatment combined with exposure to aspirin but this

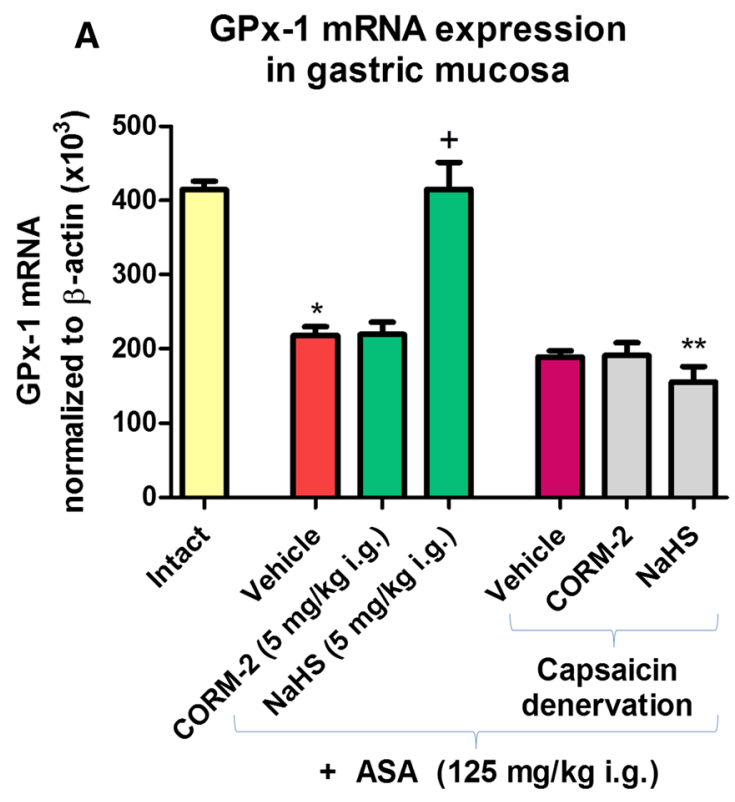

Fig. 4 Messenger RNA ( $m R N A)$ expression of a glutathione peroxidase $1(G P x-1)$ and b) superoxide dismutase $2(S O D-2)$ in gastric mucosa of rats with or without capsaicin-induced denervation and pretreated with vehicle, NaHS $(5 \mathrm{mg} / \mathrm{kg}$ intragastrically), or tricarbonyldichlororuthenium(II) dimer (CORM-2;5 mg/kg intragastrically) and administered with aspirin (ASA; $125 \mathrm{mg} / \mathrm{kg}$ intragastrically). Intact refers to healthy rats without any treatment. The results are expressed as mRNA expression of GPx-1 (a) and SOD-2 (b) normalized to $\beta$-actin expression and are the
mRNA expression for both antioxidizing enzymes was significantly decreased as compared with that in the group with intact sensory nerves and pretreated with NaHS $(p<0.05)$ (Fig. 4).

\section{Effect of NOS inhibition on CORM-2- and NaHS- mediated gastroprotection against aspirin-induced gastric damage}

Figure 5 shows that CORM-2 and NaHS administered in a dose of $5 \mathrm{mg} / \mathrm{kg}$ intragastrically significantly decreased the area of aspirin-induced gastric damage (Fig. 5a) and increased GBF (Fig. 5b) as compared with vehicle treatment $(p<0.05)$, and these results are similar to the data presented in Fig. 1. When CORM-2 was administered with L-NNA, the area of gastric lesions was significantly increased as compared with that in vehicle-pretreated rats receiving CORM-2 and compromised by aspirin (control) $(p<0.05)$ but the GBF was not significantly altered in these rats (Fig. 5). Concurrent administration of NaHS and L-NNA $(20 \mathrm{mg} / \mathrm{kg}$ intraperitoneally) significantly increased the area of gastric lesions (Fig. 5a) and significantly decreased GBF (Fig. 5b) as compared with the values observed when this $\mathrm{H}_{2} \mathrm{~S}$ donor was applied without L-NNA $(p<0.05)$.

\section{B SOD-2 mRNA expression in gastric mucosa}

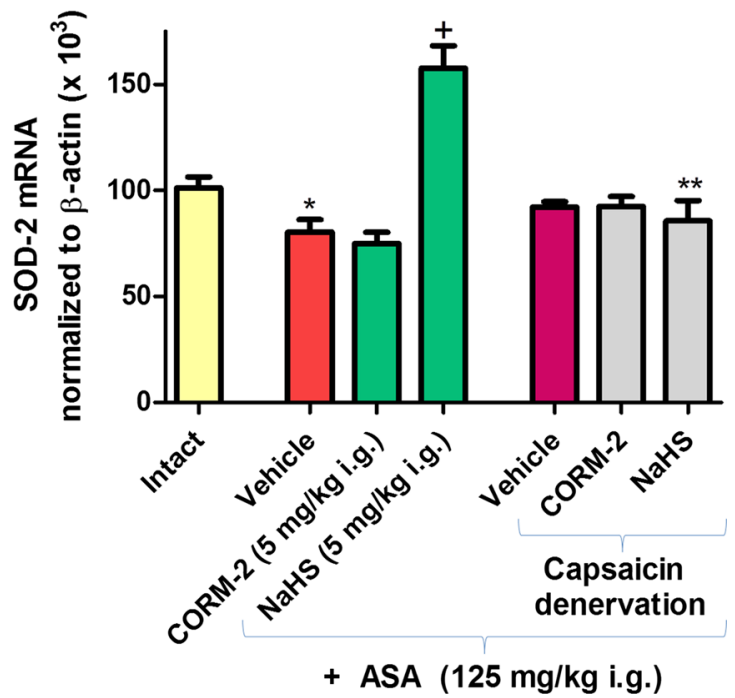

mean \pm standard error of the mean of four or five determinations per group. One asterisk indicates a significant change as compared with the values in intact gastric mucosa $(p<0.05)$. A cross indicates a significant change as compared with the values obtained in the vehicle-pretreated group with intact sensory nerves $(p<0.05)$. A significant change as compared with the values obtained in NaHSpretreated rats with intact sensory nerves is indicated by two asterisks $(p<0.05)$. i.g. intragastrically 

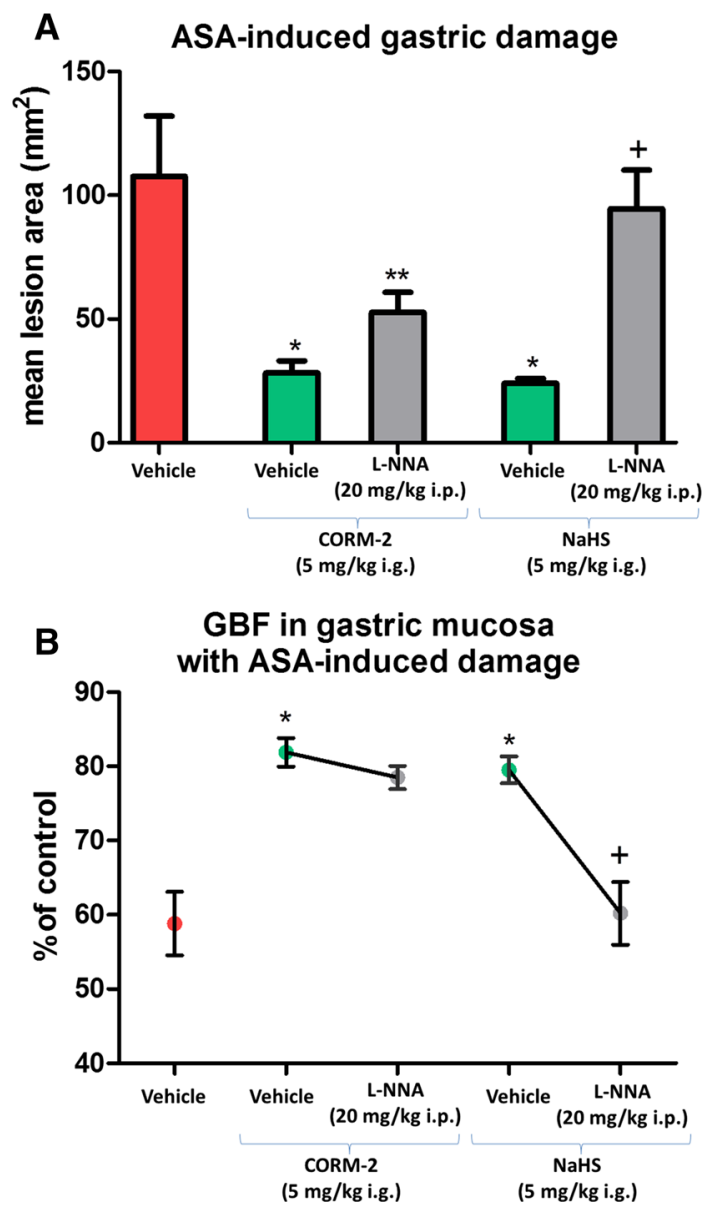

Fig. 5 a Mean lesion area and $\mathbf{b}$ gastric blood flow $(G B F)$ in gastric mucosa of rats pretreated with vehicle, NaHS $(5 \mathrm{mg} / \mathrm{kg}$ intragastrically), or tricarbonyldichlororuthenium(II) dimer (CORM-2;5 mg/kg intragastrically) alone or in combination with $N^{\mathrm{G}}$-nitro-L-arginine $(L$ $N N A ; 20 \mathrm{mg} / \mathrm{kg}$ intraperitoneally) and given aspirin (ASA; $125 \mathrm{mg} / \mathrm{kg}$ intragastrically). The results are the mean \pm standard error of the mean for four or five rats per experimental group. A significant change as compared with the respective values in the vehicle-control group is indicated by one asterisk $(p<0.05)$. A cross indicates a significant change as compared with the respective values in the NaHS-treated group $(p<0.05)$. A significant change as compared with the respective values in the group pretreated with CORM-2 alone is indicated by two asterisks $(p<0.05)$. i.g. intragastrically, i.p. intraperitoneally

\section{Protein expression of iNOS, SOD-1 and GPx-1 in gastric mucosa}

Figure 6a shows that protein expression of iNOS is significantly increased in gastric mucosa compromised by aspirin as compared with that in intact rats $(p<0.05)$. Pretreatment with CORM-2 $(5 \mathrm{mg} / \mathrm{kg}$ intragastrically) but not with $\mathrm{NaHS}(5 \mathrm{mg} / \mathrm{kg}$ intragastrically) significantly decreased iNOS protein expression as compared with that in the vehicle-control group $(p<0.05)$ (Fig. 6a). Figure 6b and $\mathrm{c}$ shows that protein expression of SOD and GPx-1 was significantly decreased in gastric mucosa in rats given aspirin as compared with intact gastric mucosa $(p<0.05)$. Pretreatment with CORM-2 (5 mg/kg intragastrically) or NaHS $(5 \mathrm{mg} / \mathrm{kg}$ intragastrically) significantly increased protein expression of GPx-1 but not SOD-1 as compared with vehicle pretreatment $(p<0.05)$ (Fig. 6b, c).

\section{Determination of MDA and 4-HNE content in gastric mucosa}

MDA and 4-HNE content in gastric mucosa was significantly increased in rats pretreated with vehicle and given aspirin as compared with intact rats $(p<0.05)$ (Fig. 7). The pretreatment with CORM-2 (5 mg/kg intragastrically) or NaHS $(5 \mathrm{mg} / \mathrm{kg}$ intragastrically) significantly decreased MDA and 4-HNE content in gastric mucosa compromised by aspirin as compared with vehicle pretreatment $(p<0.05)$.

\section{Discussion}

In this study we used an experimental model of acute aspirin-induced gastric damage, which resembles microbleedings and gastric erosions in human gastric mucosa. Previous studies have documented that the mechanism of aspirin-induced gastric damage involves an inhibition of cytoprotective prostaglandin $\mathrm{E}_{2}$ production via $\mathrm{COX}-1 /$ COX-2 enzymatic activity and the prominent fall in GBF leading to the formation of severe hemorrhagic lesions of gastric mucosa [5, 29-33]. On the other hand, recent studies reported that the endogenous gaseous mediators $\mathrm{H}_{2} \mathrm{~S}, \mathrm{CO}$, and $\mathrm{NO}$ contribute to the maintenance of gastric mucosal integrity and prevent the formation of gastric mucosal lesions induced by various noxious stimuli, such as exposure to stress or administration of ethanol, bisphosphonates, and NSAIDs, including aspirin $[5,17,19,21,22,26,34]$.

We have observed that pretreatment with CORM-2 or $\mathrm{NaHS}$, both applied in a dose of $5 \mathrm{mg} / \mathrm{kg}$, protected gastric mucosa against aspirin-induced gastric damage and increased the GBF, which is similar to what our group presented elsewhere [17]. Herein, we have demonstrated for the first time that this beneficial protective effect of an $\mathrm{H}_{2} \mathrm{~S}$ donor but not that of a $\mathrm{CO}$ donor was reduced in rats with capsaicin-induced denervation, suggesting that the protective activity of NaHS releasing $\mathrm{H}_{2} \mathrm{~S}$ unlike CORM-2 releasing $\mathrm{CO}$ can be mediated by neuropeptides such as CGRP released from sensory afferent nerves. Moreover, we found that neither NaHS nor CORM-2 had any significant influence on the gastric mucosal expression of CGRP in rats without sensory innervation treated with aspirin. However, this expression of CGRP was dramatically upregulated in rats with capsaicin-induced afferent sensory 
A iNOS protein expression

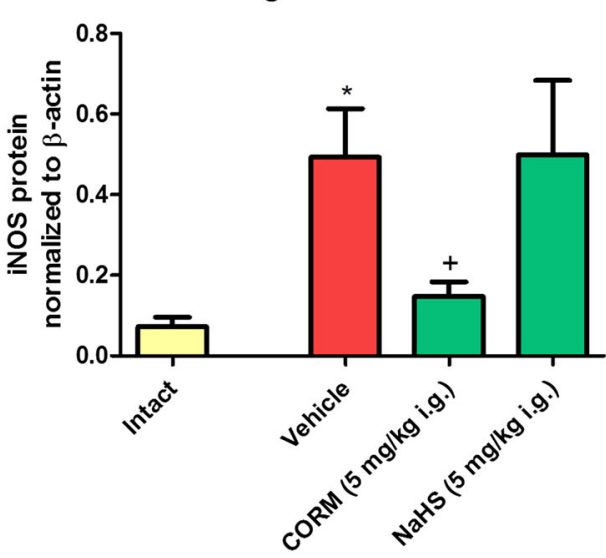

+ ASA (125 mg/kg i.g.)

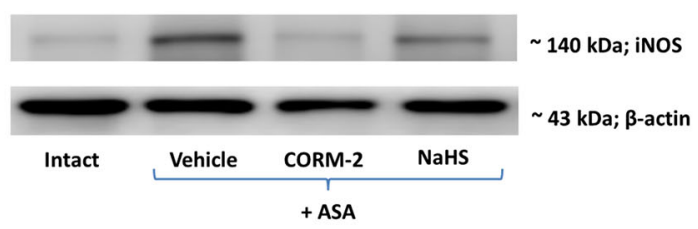

B
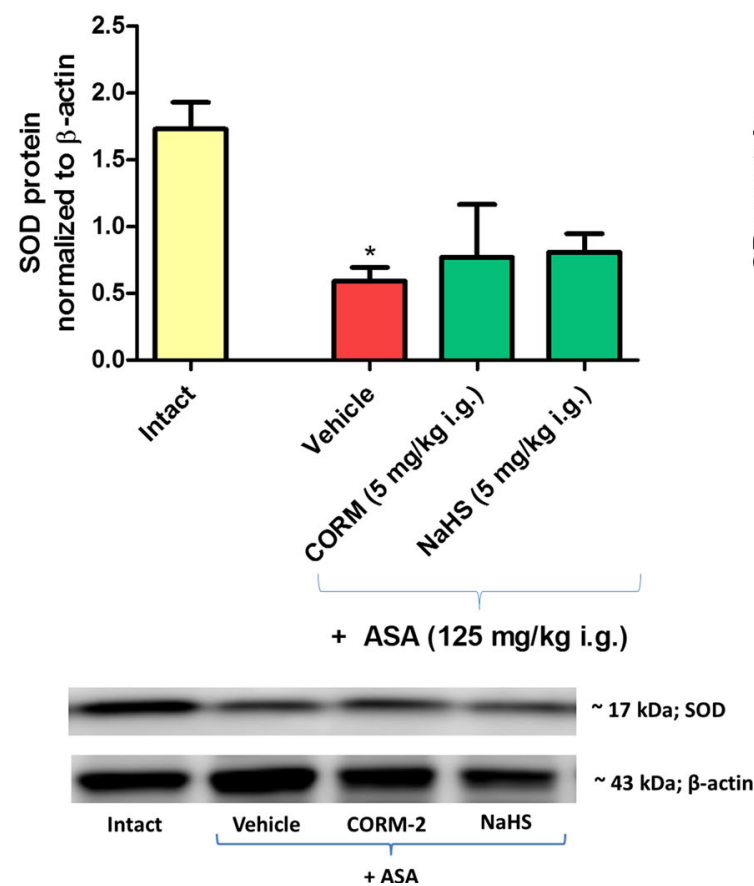

Fig. 6 Protein expression of a inducible nitric oxide synthase $i N O S$ ), b superoxide dismutase $1(S O D)$, and c glutathione peroxidase 1 $(G P x)$ in gastric mucosa of rats pretreated with vehicle, NaHS $(5 \mathrm{mg} /$ $\mathrm{kg}$ intragastrically) or tricarbonyldichlororuthenium(II) dimer (CORM-2; $5 \mathrm{mg} / \mathrm{kg}$ intragastrically) and $30 \mathrm{~min}$ later given aspirin (ASA; $125 \mathrm{mg} / \mathrm{kg}$ intragastrically). Intact refers to healthy rats without any treatment. The results are expressed as protein expression of

\section{C \\ GPx protein expression in gastric mucosa}
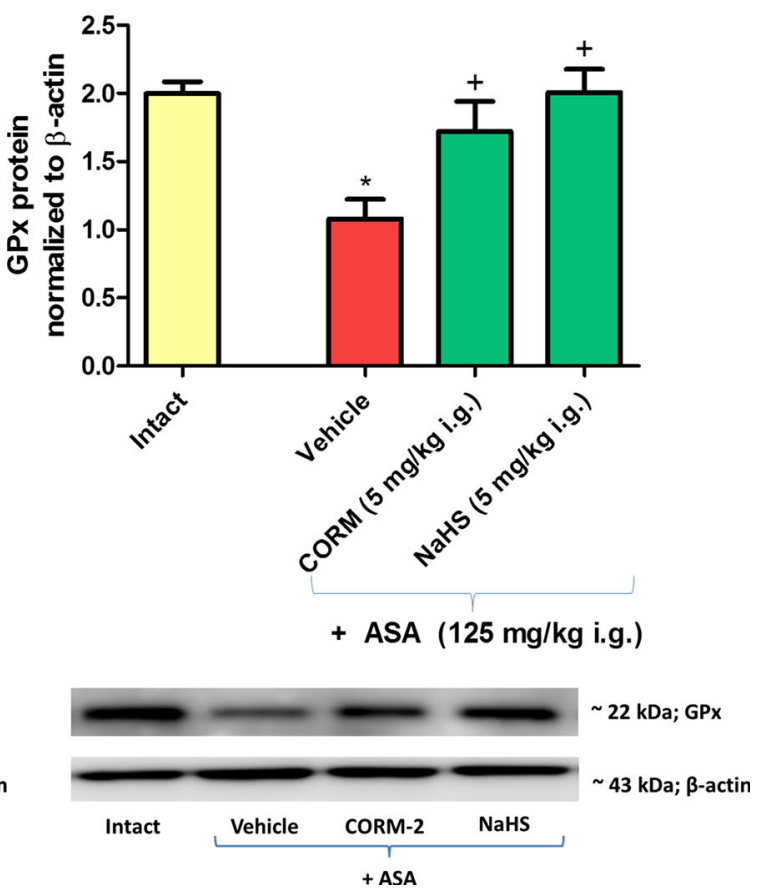

iNOS, SOD, or GPx normalized to $\beta$-actin expression and are the mean \pm standard error of the mean for four or five rats per group. An asterisk indicates a significant change as compared with the values in intact gastric mucosa $(p<0.05)$. A cross indicates a significant change as compared with the values obtained in the vehicle-pretreated group $(p<0.05)$. i.g. intragastrically 


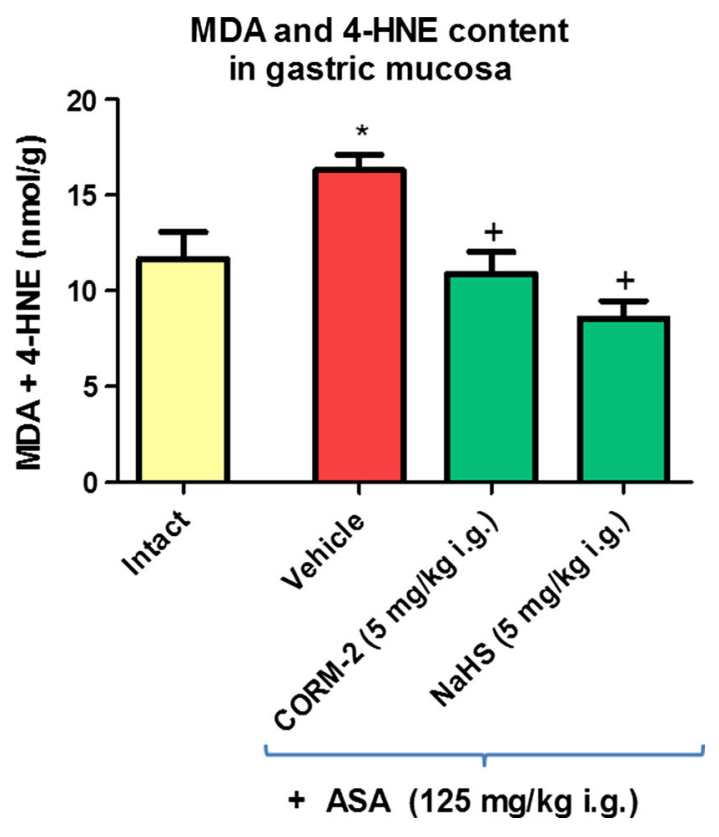

Fig. 7 Malondialdehyde (MDA) and 4-hydroxynonenal (4-HNE) content in gastric mucosa of rats pretreated with vehicle, NaHS ( $5 \mathrm{mg} / \mathrm{kg}$ intragastrically), or tricarbonyldichlororuthenium(II) dimer (CORM-2; $5 \mathrm{mg} / \mathrm{kg}$ intragastrically) and given aspirin (ASA; $125 \mathrm{mg} /$ $\mathrm{kg}$ intragastrically). Intact refers to healthy rats without any treatment. The results are the mean \pm standard error of the mean for four or five rats per group. An asterisk indicates a significant change as compared with the values in intact gastric mucosa $(p<0.05)$. A cross indicates a significant change as compared with the values obtained in the vehicle-pretreated group $(p<0.05)$. i.g. intragastrically

nerve denervation, and this effect was attenuated by pretreatment with CORM-2 but not NaHS.

We studied whether the protection by $\mathrm{H}_{2} \mathrm{~S}$ against aspirin ulcerogenesis could be mediated by the NOTRPV1-CGRP signaling pathway. Recently, the $\mathrm{H}_{2} \mathrm{~S}$ evoked vasodilatory effects were attributed to NO production and activation of the HNO-transient receptor potential cation channel A1 (TRPA1)-CGRP pathway, indicating that these mediators can contribute to the enhancement in the gastric microcirculation observed in the present study [35]. When an $\mathrm{H}_{2} \mathrm{~S}$ donor was administered in combination with the TRPV1 antagonist capsazepine, the NaHS-induced protection was reversed. However, capsazepine did not affect the NaHS-induced increase in GBF, suggesting that in this model $\mathrm{H}_{2} \mathrm{~S}$ can regulate GBF with no involvement of TRPV1 receptors. On the other hand, capsazepine failed to influence the CORM-2-induced attenuation of aspirin damage and the accompanying rise in GBF. We observed that capsaicininduced denervation even further enhanced the increase of mRNA expression of proinflammatory markers such as iNOS, IL-1 $\beta$, COX-2, and HO- 1 observed in rats subjected to aspirin. The expression of proinflammatory IL- $1 \beta$ mRNA was abrogated by CORM-2, and this effect was not influenced by capsaicin-induced denervation in contrast to groups pretreated with NaHS. However, neither a $\mathrm{CO}$ donor nor $\mathrm{H}_{2} \mathrm{~S}$-releasing $\mathrm{NaHS}$, decreased upregulated mRNA expression of HO-1, iNOS, or COX-2 in rats with sensory nerve ablation. On the basis of these findings, we assume that $\mathrm{CO}$ decreased infiltration of IL- $1 \beta$-induced activation of neutrophils and macrophages in the gastric mucosa after administration of aspirin in rats with capsaicin-induced denervation. However, this gaseous mediator was not sufficiently effective in regulation of intracellular inflammation because it failed to affect an increase in iNOS, HO-1, and COX-2 mRNA expression in these rats with capsaicin-induced denervation. In contrast to rats with intact sensory nerves, the pretreatment with NaHS did not alter the mRNA expression of the antioxidative enzymes GPx-1 or SOD-2 in gastric mucosa compromised by aspirin, which was evidently suppressed in rats with capsaicin-induced denervation, confirming that $\mathrm{H}_{2} \mathrm{~S}$-donor-mediated gastroprotection depends on afferent sensory nerve activity. The results of the present study corroborate with previously published evidence that NaHS-induced gastroprotection against stress-induced gastric mucosal lesions is attenuated in rats with functional ablation of afferent sensory nerves [19]. Therefore, we conclude that the gastroprotective effect of NaHS releasing $\mathrm{H}_{2} \mathrm{~S}$ against aspirin-induced lesions depends on the activity of afferent sensory neurons and CGRP, which is known to exert vasodilatory, anti-inflammatory, and antioxidative properties. However, sensory nerves releasing vasodilatory neuropeptides seem to be less important in CORM-2-induced gastroprotection because despite this $\mathrm{CO}$ donor reduced aspirin-induced damage, it failed to attenuate all molecular proinflammatory and antioxidative parameters overexpressed in rats with capsaicin-induced denervation.

Our data suggest that $\mathrm{H}_{2} \mathrm{~S}$-induced gastroprotection against aspirin-induced damage may involve endogenous NO biosynthesis since administration of a nonselective NO inhibitor, L-NNA, completely abolished the protective and hyperemic effects of NaHS in rats administered with aspirin. CO-mediated gastroprotection was only reduced when CORM-2 was combined with L-NNA. Moreover, the upregulation of gastric mucosal iNOS protein expression induced by aspirin was decreased in rats pretreated with CORM-2 but not with NaHS. This finding is in keeping with our previous observation that $\mathrm{CO}$ released from CORM-2 decreased NO content that was possibly elevated because of an increase in iNOS activity in gastric mucosa of rats compromised by stress and that L-NNA had no effect on the gastroprotective action of this compound [26]. Regarding the effect of L-NNA on NaHS-induced gastroprotection, there is convincing evidence that $\mathrm{H}_{2} \mathrm{~S}$ can interact with $\mathrm{NO}$ in the regulation of mucus secretion, $\mathrm{GBF}$, and gastric mucosal defense in experimental models 
and in regulation of vascular tone in animals and humans $[36,37]$. We cannot exclude the possibility that $\mathrm{NO}$ is involved in CORM-2-induced gastroprotection because an in vitro study revealed that CORM-2 directly enhanced endothelial NOS activity mediated by intracellular calcium release, intracellular Akt signaling, and endothelial NOS dimerization [38]. Therefore, we conclude that endogenous NO can mediate $\mathrm{H}_{2} \mathrm{~S}$ gastroprotection against aspirin-induced gastric damage and that this endogenous mediator can be, only in part, involved in CO-induced gastroprotection.

After administration of aspirin, lipid peroxidation was increased since the MDA and 4-HNE content in gastric mucosa reached higher concentrations than those recorded in healthy intact rats, whereas the protein expression and mRNA expression in gastric mucosa for the antioxidative enzymes GPx-1 and SOD were both decreased in aspirintreated gastric mucosa. CORM-2 and NaHS prevented an aspirin-induced increase in gastric mucosal lipid peroxidation and restored protein expression of GPx-1 but not that of SOD-1. This effect could be explained by an aspirin-induced decrease in gastric mucosa of the availability of metals, which are known to be bound to SOD $[39,40]$. Both CORM-2 and NaHS failed to reduce this effect of aspirin, because SOD protein expression remained decreased as observed $1 \mathrm{~h}$ after aspirin administration. We speculate that a longer time after aspirin administration than that used in our study is needed to observe the influence of NaHS and CORM-2 on aspirin-induced impairment in SOD protein expression. However, in contrast to CORM-2, pretreatment with NaHS increased mRNA expression for antioxidative SOD-2 in gastric mucosa compromised by aspirin. NaHS but not CORM- 2 restored GPx-1 mRNA expression in gastric mucosa injured by aspirin. Together these observations suggest that mainly $\mathrm{H}_{2} \mathrm{~S}$ but to lesser degree of $\mathrm{CO}$ can promote an antioxidative response in gastric mucosa at the mRNA level for both enzymes. It has previously been reported that differences in GPx-1 and SOD activities could serve as a marker of the pathological condition [41]. Our present observations support recent findings that $\mathrm{H}_{2} \mathrm{~S}$-releasing Lawesson's reagent or another $\mathrm{CO}$ donor, dimanganese decacarbonyl, protected gastric mucosa against alendronate-induced gastric damage via regulation of oxidative stress as manifested by an increase in reduced glutathione content and a decrease in MDA content in rat gastric mucosa injured by alendronate $[42,43]$. Thus, we conclude that the antioxidative properties of the gaseous mediators $\mathrm{CO}$ and $\mathrm{H}_{2} \mathrm{~S}$ can contribute, at least in part, to the observed gastroprotective effect of these molecules against aspirininduced gastric damage. $\mathrm{CO}$ seems to protect gastric mucosa against oxidation induced by aspirin, whereas additionally $\mathrm{H}_{2} \mathrm{~S}$ stimulates an antioxidative gastric mucosal response at the molecular level.

From the findings taken together, we conclude that $\mathrm{H}_{2} \mathrm{~S}$-mediated gastroprotection against aspirin-induced mucosal lesions involves an increase in gastric microcirculation mediated by endogenous NO. However, a COmediated beneficial protective effect against aspirin-induced damage may depend only in part on NO biosynthesis. NaHS-induced gastroprotection unlike CORM-2induced gastroprotection depends on the activity of afferent sensory nerves releasing CGRP and on the activation of TRPV1. Both $\mathrm{H}_{2} \mathrm{~S}$ released from NaHS and $\mathrm{CO}$ released from CORM-2 attenuate the aspirin-induced rise in MDA content considered as an index of gastric mucosal lipid peroxidation and can improve the antioxidative status of gastric mucosa because of the restoration of antioxidative GPx-1 protein expression impaired by aspirin treatment.

\section{Compliance with ethical standards}

Conflict of interest The authors declare that they have no conflict of interest.

Open Access This article is distributed under the terms of the Creative Commons Attribution 4.0 International License (http://crea tivecommons.org/licenses/by/4.0/), which permits unrestricted use, distribution, and reproduction in any medium, provided you give appropriate credit to the original author(s) and the source, provide a link to the Creative Commons license, and indicate if changes were made.

\section{References}

1. Kauffman G. Aspirin-induced gastric mucosal injury: lessons learned from animal models. Gastroenterology. 1989;96:606-14.

2. Hudson N, Hawkey CJ. Non-steroidal anti-inflammatory drug associated upper gastrointestinal ulceration and complications. Eur J Gastroenterol Hepatol. 1993;5:412-9.

3. Bjarnason I, Scarpignato C, Takeuchi K, et al. Determinants of the short-term gastric damage caused by NSAIDs in man. Aliment Pharmacol Ther. 2007;26(1):95-106.

4. Sostres C, Lanas A. Gastrointestinal effects of aspirin. Nat Rev Gastroenterol Hepatol. 2011;8:385-94. doi:10.1038/nrgastro. 2011.97.

5. Fiorucci S, Antonelli E, Distrutti E, et al. Inhibition of hydrogen sulfide generation contributes to gastric injury caused by antiinflammatory nonsteroidal drugs. Gastroenterology. 2005;129:1210-24.

6. Magierowski M, Jasnos K, Kwiecien S, et al. Role of hydrogen sulfide in the physiology of gastrointestinal tract and in the mechanism of gastroprotection. Postepy Hig Med Dosw. 2013;67:150-6.

7. Farrugia G, Szurszewski JH. Carbon monoxide, hydrogen sulfide, and nitric oxide as signaling molecules in the gastrointestinal tract. Gastroenterology. 2014;147:303-13.

8. Jasnos K, Magierowski M, Kwiecien S, Brzozowski T. Carbon monoxide in human physiology-its role in the gastrointestinal tract. Postepy Hig Med Dosw. 2014;68:101-9. 
9. Flannigan KL, Agbor TA, Blackler RW, et al. Impaired hydrogen sulfide synthesis and IL-10 signaling underlie hyperhomocysteinemia-associated exacerbation of colitis. Proc Natl Acad Sci U S A. 2014;111(37):13559-64. doi:10.1073/pnas.1413390111.

10. Magierowski M, Magierowska K, Kwiecien S, et al. Gaseous mediators nitric oxide and hydrogen sulfide in the mechanism of gastrointestinal integrity, protection and ulcer healing. Molecules. 2015;20(5):9099-123.

11. Jin Z, Chan $H$, Ning J, et al. The role of hydrogen sulfide in pathologies of the vital organs and its clinical application. J Physiol Pharmacol. 2015;66(2):169-79.

12. Gyires K, Toth VE, Zadori ZS. Gastric mucosal protection: from the periphery to the central nervous system. J Physiol Pharmacol. 2015;66(3):319-29.

13. Romerowicz-Misielak M, Oren DA, Sowa-Kucma M, et al. Changes in melatonin synthesis parameters after carbon monoxide concentration increase in the cavernous sinus. J Physiol Pharmacol. 2015;66(4):505.

14. Wallace JL, Caliendo G, Santagada V, et al. Markedly reduced toxicity of a hydrogen sulphide-releasing derivative of naproxen (ATB-346). Br J Pharmacol. 2010;159(6):1236-46. doi:10.1111/ j.1476-5381.2009.00611.x.

15. Flannigan KL, Wallace JL. Hydrogen sulfide-based anti-inflammatory and chemopreventive therapies: an experimental approach. Curr Pharm Des. 2015;21(21):3012-22.

16. Paul-Clark M, Elsheikh W, Kirkby N, et al. Profound chemopreventative effects of a hydrogen sulfide-releasing NSAID in the APCMin/+ mouse model of intestinal tumorigenesis. PLoS ONE. 2016;11(2):e0147289. doi:10.1371/journal.pone.0147289.

17. Magierowski M, Magierowska K, Hubalewska-Mazgaj M, et al. Interaction between endogenous carbon monoxide and hydrogen sulfide in the mechanism of gastroprotection against acute aspirin-induced gastric damage. Pharmacol Res. 2016;114:235-50. doi:10.1016/j.phrs.2016.11.001.

18. Kwiecien S, Konturek PC, Sliwowski Z, et al. Interaction between selective cyclooxygenase inhibitors and capsaicin-sensitive afferent sensory nerves in pathogenesis of stress-induced gastric lesions. Role of oxidative stress. J Physiol Pharmacol. 2012;63(2):143-51.

19. Magierowski M, Jasnos K, Kwiecien S, et al. Endogenous prostaglandins and afferent sensory nerves in gastroprotective effect of hydrogen sulfide against stress-induced gastric lesions. PLoS ONE. 2015;10(3):e0118972. doi:10.1371/journal.pone.0118972.

20. Motterlini R, Clark JE, Foresti R, et al. Carbon monoxide-releasing molecules: characterization of biochemical and vascular activities. Circ Res. 2002;90:E17-24.

21. Magierowska K, Magierowski M, Hubalewska-Mazgaj M, et al. Carbon monoxide (CO) released from tricarbonyldichlororuthenium(II) dimer (CORM-2) in gastroprotection against experimental ethanol-induced gastric damage. PLoS ONE. 2015;10(10):e0140493. doi:10.1371/journal.pone.0140493.

22. Magierowski M, Magierowska K, Szmyd J, et al. Hydrogen sulfide and carbon monoxide protect gastric mucosa compromised by mild stress against alendronate injury. Dig Dis Sci. 2016;61(11):3176-89. doi:10.1007/s10620-016-4280-5.

23. Konturek PC, Brzozowski T, Engel M, et al. Ghrelin ameliorates colonic inflammation. Role of nitric oxide and sensory nerves. J Physiol Pharmacol. 2009;60:41-7.

24. Griffith OW, Kilbourn RG. Nitric oxide synthase inhibitors: amino acids. Methods Enzymol. 1996;268:375-92.

25. Kwiecien S, Ptak-Belowska A, Krzysiek-Maczka G, et al. Asymmetric dimethylarginine, an endogenous inhibitor of nitric oxide synthase, interacts with gastric oxidative metabolism and enhances stress-induced gastric lesions. J Physiol Pharmacol. 2012;63(5):515-24.

26. Magierowska K, Magierowski M, Surmiak M, et al. The protective role of carbon monoxide $(\mathrm{CO})$ produced by heme oxygenases and derived from the CO-releasing molecule CORM-2 in the pathogenesis of stress-induced gastric lesions: evidence for non-involvement of nitric oxide (NO). Int $\mathrm{J}$ Mol Sci. 2016;17(4):E442. doi:10.3390/ijms17040442.

27. Magierowski M, Jasnos K, Sliwowski Z, et al. Exogenous asymmetric dimethylarginine (ADMA) in pathogenesis of ischemia-reperfusion-induced gastric lesions: interaction with protective nitric oxide (NO) and calcitonin gene-related peptide (CGRP). Int J Mol Sci. 2014;15(3):4946-64. doi:10.3390/ ijms15034946.

28. Schmittgen TD, Livak KJ. Analyzing real-time PCR data by the comparative CT method. Nat Protoc. 2008;3:1101-8. doi:10. 1038/nprot.2008.73.

29. Okabe S, Takeuchi K, Nakamura K, et al. Pathogenesis of gastric lesions induced by aspirin in the pylorus-ligated rat. Jpn J Pharmacol. 1974;24(3):363-71.

30. Konturek SJ, Brzozowski T, Piastucki I, et al. Prevention of ethanol and aspirin-induced gastric mucosal lesions by paracetamol and salicylate in rats: role of endogenous prostaglandins. Gut. 1982;23(6):536-40.

31. Perretti M, Mugridge KG, Wallace JL, et al. Reduction of aspirininduced gastric damage in rats by interleukin-1 beta: possible involvement of endogenous corticosteroids. J Pharmacol Exp Ther. 1992;261(3):1238-47.

32. Pongpiriyadacha Y, Matsuda H, Morikawa T, et al. Protective effects of polygodial on gastric mucosal lesions induced by necrotizing agents in rats and the possible mechanisms of action. Biol Pharm Bull. 2003;26(5):651-7.

33. Zhang JY, Wu QF, Wan Y, et al. Protective role of hydrogen-rich water on aspirin-induced gastric mucosal damage in rats. World $\mathrm{J}$ Gastroenterol. 2014;20(6):1614-22. doi:10.3748/wjg.v20.i6. 1614.

34. Wallace JL, Caliendo G, Santagada V, et al. Gastrointestinal safety and anti-inflammatory effects of a hydrogen sulfide-releasing diclofenac derivative in the rat. Gastroenterology. 2007;132(1):261-71.

35. Eberhardt M, Dux M, Namer B, et al. $\mathrm{H}_{2} \mathrm{~S}$ and $\mathrm{NO}$ cooperatively regulate vascular tone by activating a neuroendocrine HNOTRPA1-CGRP signalling pathway. Nat Commun. 2014;5:4381. doi:10.1038/ncomms5381.

36. Lucetti LT, Silva RO, Santana AP, et al. Nitric oxide and hydrogen sulfide interact when modulating gastric physiological functions in rodents. Dig Dis Sci. 2016. doi:10.1007/s10620-0164377-x.

37. Materazzi S, Zagli G, Nassini R, et al. Vasodilator activity of hydrogen sulfide $\left(\mathrm{H}_{2} \mathrm{~S}\right)$ in human mesenteric arteries. Microvasc Res. 2016;109:38-44. doi:10.1016/j.mvr.2016.11.001.

38. Yang PM, Huang YT, Zhang YQ, et al. Carbon monoxide releasing molecule induces endothelial nitric oxide synthase activation through a calcium and phosphatidylinositol 3-kinase/ Akt mechanism. Vascul Pharmacol. 2016. doi:10.1016/j.vph. 2016.09.010.

39. Richardson J, Thomas KA, Rubin BH, et al. Crystal structure of bovine $\mathrm{Cu}, \mathrm{Zn}$ superoxide dismutase at $3 \mathrm{~A}$ resolution: chain tracing and metal ligands. Proc Natl Acad Sci U S A. 1975;72(4):1349-53.

40. Wang X. A chelate theory for the mechanism of action of aspirinlike drugs. Med Hypotheses. 1998;50(3):239-51. 
41. Park EM, Ramnath N, Yang GY, et al. High superoxide dismutase and low glutathione peroxidase activities in red blood cells predict susceptibility of lung cancer patients to radiation pneumonitis. Free Radic Biol Med. 2007;42(2):280-7.

42. Nicolau LA, Silva RO, Damasceno SR, et al. The hydrogen sulfide donor, Lawesson's reagent, prevents alendronate-induced gastric damage in rats. Braz J Med Biol Res. 2013;46:708-14.
43. Costa NR, Silva RO, Nicolau LA, et al. Role of soluble guanylate cyclase activation in the gastroprotective effect of the $\mathrm{HO}-1 / \mathrm{CO}$ pathway against alendronate-induced gastric damage in rats. Eur J Pharmacol. 2013;700:51-9. 